\title{
O FUMO ENTRE DOCENTES E DISCENTES DAS ESCOLAS DE ENFERMAGEM
}

\author{
Maria Apparecida Valente* \\ Ana Maria Kazue Miyadahira* \\ Cilene Aparecida Costardi Ide** \\ Edna Ikumi Umebayashi Takahashi * \\ Eliane Corrêa Chaves *: \\ Katia Grillo Padilha*: \\ Miako Kimura**
}

VALENTE, M. A.; MIYADAHIRA, A. M. K.; IDE, C. A. C.; TAKAHASHI, E. I. U.; CHAVES, E. C.; PADILHA, K. G.; KIMURA, M. O fumo entre docentes e discentes das Escolas de Enfermagem. Rev. Esc. Enf. USP, São Paulo, 16(2)147-163, 1982.

As autoras, no presente trabalho, apresentam o resultado de um levantamento, feito entre docentes e discentes de três escolas do curso de graduação em enfermagem do Município de São Paulo, sobre o hábito de fumar, para verificar freqüência de fumantes, ex-fumantes e não fumantes e causas que os levaram ao hábito de fumar $e$ a abandoná-lo. Na população de 636 estudantes, encontrou-se $24,1 \%$ fumantes, $68,5 \%$ não fumantes e 7,4\% ex-fumantes e, entre 117 docentes, $23,1 \%, 62,4 \%$ e $14,5 \%$-respectivamente. A causa mais freqüente que levou ao hábito de fumar, em ambos os grupos, foi curiosidade $(35,2 \%$ e $28,2 \%$ respectivamente) e ao abandono foi a consciência sobre os maleficios do fumo para a saúde $(72,5 \%$ e $65,2 \%$ respectivamente); este foi o principal motivo dos não fumantes nunca terem iniciado o hábito de fumar. Foram estudados também a idade em que se iniciou o hábito de fumar, o número de cigarros fumados por dia, os efeitos do tabagismo sobre as pessoas e a orientaçâo dada a estas sobre os danos do fumo para a saúde.

\section{INTRODUÇÃO}

O hábito de fumar foi introduzido no continente europeu pelos colonizadores que, após a descoberta da América, tendo contato com os indigenas da região, conheceram e aderiram, progressivamente, ao uso da planta do tabaco.

No seu início, a planta era conhecida e utilizada como erva que possuía virtudes terapêuticas, sendo empregada no tratamento de vários males. Com o tempo, foi verificada a inexistência dos efeitos terapêuticos do tabaco, porém, o hábito de fumar já havia sido difundido na Europa e atingido a Ásia e a Âfrica (ROSEMBERG, 1977).

* Professor Assistente do Departamento de Enfermagem Médico-Cirúrgica da Escola de Enfermagem da USP - disciplin:a Enfermagem Médico-Cirúrgica. Mestre em Enfermagem. Enfermeira.

* Auxiliar de Ensino do Departamento de Enfermagem Médico-Cirúrgica da Escola de Enfermagem da USP - disciplina Enfermagem Médico-Cirúrgica. Enfermeira. 
Em fins do século XVI, o comércio do tabaco já era altamente lucrativo, dando origem a grandes potências industriais. Do século XVII ao século XIX, o tabaco teve repercussão principalmente em duas diferentes formas de uso, o cachimbo e o charuto. No entanto, uma nova modalidade de fumar, o cigarro, foi conquistando as populações e tornando-se um dos maiores valores do comércio internacional no presente século (ROSEMBERG, 1977).

O tabagismo, sem dúvida alguma, tornou-se em nossos dias um dos hábitos mais disseminados pelo mundo, atingindo larga faixa etária, com predominância no jovem, coexistindo, com a iniciação desse hábito, fatores sociais e psicológicos.

Para ROSEMBERG (1977), as razões psicológicas e sociológicas que levam o jovem ao tabagismo variam segundo a atitude e o comportamento do grupo ao qual se integra, da própria família e do próprio indivíduo. As justificativas mais freqüentemente relacionadas ao hábito de fumar são de que o cigarro é uma forma de contestação ou auto-afirmação para o jovem, um símbolo de independência ou rebeldia, um elemento de comunicação com o amigo, um disfarce para a timidez, etc. (GRAY, 1980; RESOURCE GUIDE, 1976; ROSEMBERG, 1977).

Além desses fatores psicológicos e sociais, ROSEMBERG discute a existência de razões genéticas, antropológicas e outras.

Em nosso meio, no entanto, quaisquer que sejam os fatores envolvidos no hábito de fumar, os mesmos tornam-se supérfluos em face do estrondoso efeito massificante da propaganda de cigarros, cujo conteúdo visa especificamente sensibilizar os adolescentes, uma vez que dão sempre a conotação de sucesso, "status"; por ser transmitida principalmente pela televisão, atinge rapidamente a coletividade.

Por mais que a literatura médica e não médica demonstrem cientificamente os graves prejuizos do cigarro para a saúde (GODBER, 1970; HORN, 1975; RESOURGE GUIDE, 1976; ROSEMBERG, 1977; SCHURER, 1980; WILLIARD, 1979), tais dados parecem não atingir diretamente o fumante brasileiro e muito menos a liderança política de nosso país, uma vez que cerca de cinqüenta (50) projetos de lei contra o fumo tramitam há mais de dez anos no Senado e na Câmara Federal (AUGELLI, 1981).

No Brasil, o cigarro é considerado como uma riqueza nacional; entretanto o fumo é responsável por gastos incalculáveis em consultas médicas, internações hospitalares, pensões por invalidez e morte, além de ser agente direto e indireto de pelo menos vinte e seis (26) doenças exaustivamente pesquisadas (AUGELLI, 1981).

Ciente de que tais conseqüências atingem grande número de países e a fim de fortalecer a consciência da séria ameaça à saúde que constitui o fumo, a Organização Mundial de Saúde (OMS) lançou, no dia Mundial da Saúde, em 1980 (7 de abril) o seguinte tema: "Fumo ou 
Saúde: a escolha é sua". A mesma Organização, desde 1970, vem trabalhando intensamente no combate ao hábito de fumar, incentivando as naçōes quanto ao estabelecimento de medidas que visam o controle desse hábito, através da promulgação de leis necessárias, campanhas de educação à saúde e suspensão de propaganda favorável ao fumo. No entanto, em nosso país, somente em 1981 foi estabelecida uma medida legislativa (Lei 2.845, de 20 de maio de $1981^{*}$ ) proibindo o fumo em alguns locais específicos das unidades escolares, das praças esportivas e de outros estabelecimentos como por exemplo os hospitais.

Acreditamos, porém, ser necessário o estabelecimento de outras medidas mais restritivas e, associada a elas, uma campanha maciça de educação à saúde, a fim de que possa ser obtido efeito muito mais definitivo na população.

Como profissionais da saúde que somos, sentimos que o nosso papel de educador assume importância vital frente a esse problema. $\mathrm{Na}$ prática, deparamos, com freqüência, situações em que é necessária orientação criteriosa e precisa sobre os maleficios do fumo, em que a mesma é feita, por profissionais que não demonstram muita conviç̧ão ou que são desacreditados por serem fumantes ou por estarem com vestígios de cigarro.

Nós, como docentes de uma Escola de Enfermagem, responsáveis não só por toda a assistência de enfermagem prestada aos pacientes pelos alunos, como também pela formação destes, acreditamos ser necessário reexame da nossa postura frente a esse problema.

Estamos devidamente conscientizadas dos prejuizos do fumo para a saúde? Transmitimos isso para os nossos alunos? Mesmo sendo fumantes, tratamos do assunto com a seriedade merecida?

Considerando que: a) nos curriculos dos cursos de graduação em Enfermagem dificilmente são abordados com destaque os efeitos do fumo na saúde e, b) para orientar aqueles que estão sob seus cuidados no hospital e na comunidade a enfermeira tem obrigação de educar-se sobre o fumo e seus efeitos (SCHURER, 1980), resolvemos fazer um estudo nas Escolas de Enfermagem do Município de São Paulo, a fim de caracterizar o hábito de fumar entre docentes e discentes do curso de graduação. Para isso, os seguintes objetivos foram propostos:

1. Verificar a freqüência de fumantes, ex-fumantes e não fumantes entre docentes e discentes das Escolas de Enfermagem do Município de São Paulo.

2. Verificar as causas que induziram essas pessoas a adquirir o hábito de fumar, deixar de fumar ou não fumar.

3. Verificar a opinião dos 2 grupos a respeito dos prejuízos do fumo para a saúde.

4. Verificar se os docentes e discentes aplicam estes conhecimentos dando orientação às pessoas com as quais se relacionam.

* Diário Ofjcial do Estado de São Paulo de 21 de maio de 1982. 


\section{METODOLOGIA}

O presente trabalho abrangeu para estudo os cursos de Graduação das Escolas de Enfermagem do Município de São Paulo que tivessem turmas de alunos cursando regularmente todos os anos do curso de graduação. Por esse motivo, foi excluída a Escola de Enfermagem da Zona Leste que se encontrava em seu primeiro ano de funcionamento. A Faculdade Adventista de Enfermagem não foi, também, incluída no Estudo, por ser proibido fumar nesse estabelecimento de ensino, o que poderia interferir nas respostas. Assim, participaram deste trabalho as seguintes escolas: Escola de Enfermagem da USP, Departamento de Enfermagem da Escola Paulista de Medicina e Escola de Enfermagem São José.

\section{População}

Foi constituída por todos os docentes e discentes que pertenciam às escolas relacionadas, no ano de 1981 .

\section{Coleta de dados}

1. Instrumento. $\mathrm{O}$ instrumento de coleta de dados constou de um questionário com dezessete perguntas. As pesguntas de número 1 a 5 foram referentes à identificação: da escola de enfermagem; do tipo de vinculação mantido por docentes e discentes com a mesma; e sexo e idade destas pessoas. As perguntas de número 6 a 17 foram diretamente relacionadas aos objetivos da pesquisa: destinavam-se a coleta de informações sobre docentes e alunos quanto ao hábito de fumar, ou seja, se fumantes, ex-fumantes ou não fumantes (perguntas de número 6 a 10); as causas que os levaram a tal atitude (número 11 a 13); os seus conhecimentos teóricos sobre os efeitos do fumo para a saúde (número 14 a 16); e a aplicação prática desses conhecimentos com as pessoas do seu relacionamento (número 17), ANEXO I.

2. Operacionalização da coleta de dados. A aplicação dos questionários entre os docentes e discentes das escolas em estudo foi feita durante o mês de maio de 1981, pelas próprias pesquisadoras, que contaram com a colaboração de docentes da Escola de Enfermagem São José e do Departamento de Enfermagem da Escola Paulista de Medicina. Os questionários foram distribuidos para os alunos nas salas de aula, sendo recolhidos logo depois de seu preenchimento. Para os docentes os questionários foram entregues individualmente acompanhados de uma carta, ANEXO II.

\section{RESULTADOS E COMENTARIOS}

Das três escolas escolhidas para o presente estudo, participaram $117(89,3 \%)$ docentes, de um total de 131 e $636(86,1 \%)$ discentes, de um total de 739 . Houve, portanto, 753 participantes, que constituiram $86,5 \%$ da população total, com apenas $13,4 \%$ de isenção. 
A distribuição dessa população nas diferentes escolas encontra-se na tabela 1 .

TABELA 1

Distribuição da população de acordo com a Escola de Enfermagem a que pertence.

\begin{tabular}{cccccccc}
\hline \multirow{2}{*}{$\begin{array}{c}\text { Escola de } \\
\text { Enfermagem }\end{array}$} & \multicolumn{3}{c}{ Tipo de vinculo } & \multicolumn{2}{c}{ Total } \\
\cline { 2 - 7 } & $N^{4}$ & $\%$ & $N^{4}$ & $\%$ & & \\
\hline USP & 75 & 64,1 & 309 & 48,6 & 384 & 51,0 \\
PAULISTA & 26 & 22,2 & 213 & 33,5 & 239 & 31,7 \\
SAO JOSE & 16 & 13,7 & 114 & 17,9 & 130 & 17,3 \\
\hline TOTAL & 117 & 100,0 & 636 & 100,0 & 763 & 100,0 \\
\hline
\end{tabular}

A população foi eminentemente feminina com a presença de apenas $15(2 \%)$ elementos masculinos, entre docentes $\mathrm{e}$ discentes.

Os demais dados obtidos serão apresentados nas tabelas seguintes.

\section{TABELA 2}

Distribuição da população segundo a faixa etária.

\begin{tabular}{|c|c|c|c|c|c|c|}
\hline \multirow{3}{*}{$\begin{array}{l}\text { Faixa } \\
\text { ettaria } \\
\text { (em anos) }\end{array}$} & \multicolumn{4}{|c|}{ Vinculac ${ }^{x}$ o a Escola } & \multicolumn{2}{|c|}{ Total } \\
\hline & \multicolumn{2}{|c|}{ Docentes } & \multicolumn{2}{|c|}{ Discentes } & \multirow[t]{2}{*}{$\mathbf{N}$} & \multirow{2}{*}{$\%$} \\
\hline & $\mathbf{N}^{\bullet}$ & $\%$ & $\mathbf{N}$ & $\%$ & & \\
\hline menos de 20 & 一 & - & 147 & 23,1 & 147 & 19,5 \\
\hline $20-29$ & 28 & 23,9 & 478 & 76,2 & 506 & 67,2 \\
\hline $30-39$ & 53 & 45,3 & 11 & 1,7 & 64 & 8,5 \\
\hline $40-48$ & 23 & 19,7 & - & - & 23 & 3,1 \\
\hline 50 ou mais & 13 & 11,1 & - & 一 & 13 & $\mathbf{1 , 7}$ \\
\hline TOTAL & 117 & 100,0 & 636 & 100,0 & 753 & 100,0 \\
\hline
\end{tabular}

Pela Tabela 2 verifica-se que predomina, entre os docentes, a faixa etária 30 a 39 anos $(45,3 \%)$ e, entre os discentes, a faixa etária 20 a 29 anos $(75,2 \%)$. 


\section{TABELA 3}

Distribuição da população em relação ao hábito de fumar.

\begin{tabular}{|c|c|c|c|c|c|c|}
\hline \multirow{3}{*}{$\begin{array}{l}\text { Hábito } \\
\text { fe } \\
\text { fumar }\end{array}$} & \multicolumn{4}{|c|}{ Vỉnculação à Escola } & \multicolumn{2}{|c|}{ Total } \\
\hline & \multicolumn{2}{|c|}{ Docentes } & \multicolumn{2}{|c|}{ Discentes } & \multirow[t]{2}{*}{$\mathbf{N}$} & \multirow{2}{*}{$\%$} \\
\hline & $\mathbf{N e}$ & $\%$ & Ne & $\%$ & & \\
\hline fumante & 27 & 23,1 & 153 & 24,1 & 180 & 23,9 \\
\hline não fumante & 73 & 62,4 & 436 & 68,5 & 509 & 67,6 \\
\hline ex-fumante & 17 & 14,5 & 47 & 7,4 & 64 & 8,5 \\
\hline TOTAL & 117 & 100,0 & 636 & 100,0 & 753 & 100,0 \\
\hline
\end{tabular}

Observa-se pela Tabela 3 , que os docentes $(62,4 \%)$ e a maioria dos discentes $(68,5 \%)$ não têm o hábito de fumar. Verifica-se, também, que a porcentagem de ex-fumantes entre os docentes é praticamente o dobro da porcentagem entre os discentes. Pode-se supor que este resultado esteja relacionado ao fato de que os docentes, por fumarem há mais tempo, já tenham sentido alguns sintomas nocivos ou por terem conhecimento científico quanto aos malefícios provocados pelo fumo, à própria saúde.

\section{TABELÁ 4}

Distribuição da população segundo a idade em que iniciou o hábito de fumar.

\begin{tabular}{|c|c|c|c|c|c|c|}
\hline \multirow{3}{*}{$\begin{array}{l}\text { Idade } \\
\text { (em anos) }\end{array}$} & \multicolumn{4}{|c|}{ Vinculação à Escola } & \multirow{3}{*}{ Ne } & \multirow{3}{*}{ Total } \\
\hline & \multicolumn{2}{|c|}{ Docentes } & \multicolumn{2}{|c|}{ Discentes } & & \\
\hline & $\mathbf{N}$ & $\%$ & Ne & $\%$ & & \\
\hline $10-14$ & 3 & 7,0 & $\mathbf{5 2}$ & 26,0 & 55 & 22,7 \\
\hline $15-19$ & 19 & 44,2 & 121 & 60,5 & 140 & 57,6 \\
\hline $20-24$ & 14 & 32,6 & 22 & 11,0 & 36 & 14,8 \\
\hline $25-29$ & 6 & 13,9 & 1 & 0,5 & 7 & 2,9 \\
\hline 30 ou mais & 1 & 2,3 & - & - & 1 & 0,4 \\
\hline em branco & - & - & 4 & 2,0 & 4 & 1,6 \\
\hline TOTAL & 43 & 100,0 & 200 & 100,0 & 243 & 100,0 \\
\hline
\end{tabular}


Pela Tabela 4 verifica-se que $44,2 \%$ dos docentes e $60,5 \%$ dos discentes começaram a fumar na mesma faixa etária de 15 a 19 anos. Observa-se ainda que, no grupo de docentes, a segunda maior freqüência $(32,6 \%)$ ficou compreendida entre 20 e 24 anos; este fato pode ser justificado, em parte, pela própria diferença na aceitação social do hábito de fumar entre os dois grupos.

\section{TABELA 5}

Distribuição da população segundo há quanto tempo fuma.

\begin{tabular}{|c|c|c|c|c|c|c|}
\hline \multirow{3}{*}{$\begin{array}{l}\text { Há quanto } \\
\text { tempo fuma } \\
\text { (em anos) }\end{array}$} & \multicolumn{4}{|c|}{ Vinculacão à Escola } & \multirow{2}{*}{\multicolumn{2}{|c|}{ Total }} \\
\hline & \multicolumn{2}{|c|}{ Docentes } & \multicolumn{2}{|c|}{ Discentes } & & \\
\hline & $\mathbf{N}$ & $\%$ & $\mathbf{N}$ & $\%$ & $\mathbf{N}$ & $\%$ \\
\hline menos de 1 & - & - & 8 & 5,2 & 8 & 4,4 \\
\hline $1-2$ & - & - & 20 & 13,1 & 20 & 11,2 \\
\hline $3-4$ & - & - & 45 & 29,5 & 45 & 25,0 \\
\hline $5-6$ & 1 & 3,7 & 34 & 22,2 & 35 & 19,4 \\
\hline $7-8$ & 4 & 14,8 & 27 & 17,6 & 31 & 17,2 \\
\hline $9-10$ & - & - & 13 & 8,5 & 13 & 7,3 \\
\hline $11-15$ & 8 & 29,7 & 2 & 1,3 & 10 & 5,4 \\
\hline mais de 15 & 13 & 48,1 & 2 & 1,3 & 15 & 8,4 \\
\hline em branco & 1 & 3,7 & 2 & 1,3 & 3 & 1,7 \\
\hline TOTAL & 27 & 100,0 & 153 & 100,0 & 180 & 100,0 \\
\hline
\end{tabular}

Constata-se pela Tabela 5 que $48,1 \%$ dos docentes fumam há mais de 15 anos enquanto que $29,5 \%$ dos discentes fumam de 3 a 4 anos e que $22,2 \%$ fumam de 5 a 6 anos.

É necessário lembrar que a diferença nas idades predominantes entre os docentes (30 a 39 anos) e discentes (20 a 29 anos) é de aproximadamente 10 anos, o que explica o fato dos professores fumarem há mais tempo.

\section{TABELA 6}

Distribuição da população segundo a quantidade de cigarros fumados por dia.

\begin{tabular}{crrrrrrr}
\hline \multirow{2}{*}{$\begin{array}{c}\text { Quantidade } \\
\text { de Cigarros }\end{array}$} & \multicolumn{3}{c}{ Vinculação à Escola } & \multicolumn{2}{c}{ Total } \\
& \multicolumn{2}{c}{ Docentes } & \multicolumn{2}{c}{ Discentes } & No & $\%$ \\
& $N^{\varphi}$ & $\%$ & $N^{\circ}$ & $\%$ & & \\
\hline $1-10$ & 11 & 40,7 & 112 & 73,2 & 123 & 68,3 \\
$11-20$ & 10 & 37,1 & 39 & 25,5 & 49 & 27,2 \\
$21-30$ & 3 & 11,1 & 2 & 1,3 & 5 & 2,8 \\
31 ou mais & 2 & 7,4 & - & - & 2 & 1,1 \\
em branco & 1 & 3,7 & - & - & 1 & 0,6 \\
\hline TOTAL & 27 & 100,0 & 153 & 100,0 & 180 & 100,0 \\
\hline
\end{tabular}


Pela Tabela 6 observa-se que a maior parte dos discentes $(73,2 \%)$ e dos docentes $(40,7 \%)$ fuma de 1 a 10 cigarros por dia, sendo que entre os docentes, $37,1 \%$ fumam de 11 a 20 cigarros por dia.

\section{TABELA 7}

Distribuição da população segundo os motivos pelos quais começou a fumar.

\begin{tabular}{|c|c|c|c|c|c|c|}
\hline \multirow{3}{*}{ Motivos } & \multicolumn{4}{|c|}{ Vinculação à Escola } & \multirow{2}{*}{\multicolumn{2}{|c|}{ Total }} \\
\hline & \multicolumn{2}{|c|}{ Docentes } & \multicolumn{2}{|c|}{ Discentes } & & \\
\hline & No & $\%$ & $\mathbf{N}$ & $\%$ & $\mathbf{N}^{9}$ & $\%$ \\
\hline influência familia: & 4 & 5,6 & 24 & 6,6 & 28 & 6,5 \\
\hline influência extra familiar & 16 & 22,5 & 92 & 25,5 & 108 & 25,0 \\
\hline curiosidade e desejo & 20 & 28,2 & 127 & 35,2 & 147 & 34,1 \\
\hline ajuda enfrentar tensões & 16 & 22,5 & 55 & 15,2 & 71 & 16,4 \\
\hline sentir-se adulto & 1 & 1,5 & 15 & 4,2 & 16 & $\mathbf{3 , 7}$ \\
\hline aumenta $o$ atrativo pessoal & 2 & 2,8 & 11 & 3,1 & 13 & $\mathbf{3 , 0}$ \\
\hline $\begin{array}{l}\text { desconhecimento do prejuizo } \\
\text { à saúde }\end{array}$ & 2 & 2,8 & 12 & 3,3 & 14 & 3,2 \\
\hline outros & 3 & 4,2 & 15 & 4,1 & 18 & 4,2 \\
\hline não sabe & 7 & 9,9 & 10 & 2,8 & 17 & 3,8 \\
\hline TOTAL & 71 & 100,0 & 361 & 100,0 & 432 & 100,0 \\
\hline
\end{tabular}

Pela Tabela 7 constata-se que para os discentes, os motivos mais freqüentes que os levaram a fumar foram em ordem decrescente os seguintes: curiosidade e desejo $(35,2 \%)$, influência do grupo extra familiar $(25,5 \%)$ e a citação de que o cigarro ajuda a enfrentar tensões $(15,2 \%)$. Para os docentes, o motivo mais freqüente também foi a curiosidade e desejo $(28,2 \%)$, sendo que a influência do grupo extra familiar e a citação do cigarro ajudando a enfrentar tensões, tiveram ambos a segunda maior freqüência $(22,5 \%)$.

Tais razões são coincidentes com as apontadas no estudo feito por ROSEMBERG (1980) com um grupo de docentes e discentes do Centro de Ciências Médicas e Biológicas de Sorocaba. 


\section{TABELA 8}

Distribuição da população segundo os motivos pelos quais deixou de fumar.

\begin{tabular}{|c|c|c|c|c|c|c|}
\hline \multirow{3}{*}{ Motivos } & \multicolumn{4}{|c|}{ Vinculação à Escola } & \multirow{2}{*}{\multicolumn{2}{|c|}{ Total }} \\
\hline & \multicolumn{2}{|c|}{ Docentes } & \multicolumn{2}{|c|}{ Discentes } & & \\
\hline & $\mathbf{N}$ & $\%$ & $\mathbf{N}$ & $\%$ & $\mathbf{N}$ & $\%$ \\
\hline influência religiosa & - & - & 2 & 3,4 & 2 & 2,5 \\
\hline influência do namorado & - & - & 1 & 1,7 & 1 & 1,2 \\
\hline auto-consciéncia & 15 & 65,2 & 42 & 72,5 & 57 & 70,4 \\
\hline dever profissional & - & - & 2 & 3,4 & 2 & 2,5 \\
\hline não sentir falta & 2 & 8,7 & 3 & 5,2 & 5 & 6,2 \\
\hline não sabe & - & - & 1 & 1,7 & 1 & 1,2 \\
\hline não gostou & 一 & 一 & 5 & 8,7 & 5 & 6,2 \\
\hline gravidez & 6 & 26,1 & 2 & 3,4 & 8 & 9,8 \\
\hline TOTAL & 23 & 100,0 & 58 & 100,0 & 81 & 100,0 \\
\hline
\end{tabular}

Verifica-se pela Tabela 8 que a grande maioria dos docentes $(65,2 \%)$ e dos discentes $(72,5 \%)$ justificou o abandono do hábito de fumar pelo mesmo motivo: a autoconscientização sobre os malefícios do fumo para a saúde.

\section{TABELA 9}

Distribuição da população segundo os motivos pelos quais não ingressou no tabagismo.

\begin{tabular}{|c|c|c|c|c|c|c|}
\hline \multirow{3}{*}{ Motivos } & \multicolumn{4}{|c|}{ Vinculação à Escola } & \multirow{2}{*}{\multicolumn{2}{|c|}{ Total }} \\
\hline & \multicolumn{2}{|c|}{ Docentes } & \multicolumn{2}{|c|}{ Discentes } & & \\
\hline & $\mathbf{N}$ & $\%$ & $\mathbf{N e}$ & $\%$ & $\mathbf{N e}$ & $\%$ \\
\hline nunca senti vontade & 51 & 40,1 & 271 & 32,5 & 322 & 33,5 \\
\hline $\begin{array}{l}\text { experimentei mas não gostei } \\
\text { conhecimento do prejuizo }\end{array}$ & 24 & 18,9 & 192 & 23,0 & 216 & 22,5 \\
\hline para a saúde & 28 & 22,1 & 227 & 27,3 & 255 & 26,5 \\
\hline influência familiar & 10 & 7,8 & 78 & 9,3 & 88 & 9,2 \\
\hline influência extra familiar & 3 & 2,4 & 20 & 2,4 & 23 & 2,5 \\
\hline problemas de sauide & 1 & 0,8 & 8 & 1,0 & 9 & 0,9 \\
\hline não sei & - & - & 12 & 1,4 & 12 & 1,2 \\
\hline problemas econômicos & 1 & 0,8 & 2 & 0,2 & 3 & 0,3 \\
\hline aversão & 1 & 0,8 & 9 & 1,1 & 10 & 1,1 \\
\hline não acha razão & 4 & 3,1 & 5 & 0,6 & 9 & 0,9 \\
\hline medo da dependéncia & 1 & 0,8 & 1 & 0,1 & 2 & 0,2 \\
\hline em branco & 3 & 2,4 & 9 & 1,1 & 12 & 1,2 \\
\hline TOTAL & 127 & 100,0 & 834 & 100,0 & $96:$ & 100,0 \\
\hline
\end{tabular}


Observa-se pela Tabela 9 que os motivos mais freqüentes que levaram os dois grupos a não ingressarem no tabagismo são os mesmos e na mesma ordem: em primeiro lugar por não terem sentido vontade, em segundo lugar pelos conhecimentos dos prejuízos para a saúde e em terceiro lugar por terem experimentado, mas não terem gostado.

TABELA 10

Distribuição da população segundo a opinião sobre os efeitos prejudiciais do fumo para a saúde.

\begin{tabular}{|c|c|c|c|c|c|c|}
\hline \multirow{3}{*}{ Efeitos } & \multicolumn{4}{|c|}{ Vinculacăo à Escola } & \multirow{3}{*}{\multicolumn{2}{|c|}{ No Total }} \\
\hline & \multicolumn{2}{|c|}{ Docentes } & \multicolumn{2}{|c|}{ Discentes } & & \\
\hline & $\mathbf{N}^{\mathbf{o}}$ & $\%$ & $\mathbf{N}^{9}$ & $\%$ & & \\
\hline não traz & 2 & 1,7 & 16 & 2,5 & 18 & 2,4 \\
\hline $\operatorname{traz}$ & 106 & 90,6 & 605 & 95,1 & 711 & 94,4 \\
\hline em branco & 9 & 7,7 & 15 & 2,4 & 24 & 3,2 \\
\hline TOTAL & 117 & 100,0 & 636 & 100,0 & 753 & 100,0 \\
\hline
\end{tabular}

Pela Tabela 10 verifica-se que a maioria dos discentes $(95,1 \%)$ e dos docentes $(90,6 \%)$ considera que o fumo traz prejuízo para a saúde. Entre os efeitos nocivos do fumo citados pelos discentes predominam os seguintes: problemas respiratórios $(36,8 \%)$, aumento da incidência de câncer $(17,6 \%)$, problemas cardiovasculares $(12,6 \%)$ distúrbios orgânicos generalizados $(9,4 \%)$ e dependência $(6,0 \%)$. Os docentes citam os seguintes efeitos do fumo sobre a saúde: problemas respiratórios $(32,6 \%)$, alterações cardiovasculares $(20,7 \%)$, aumento da incidência de câncer $(14,3 \%)$, distúrbios orgânicos generalizados $(8,0 \%)$ e prejuízos durante a gravidez $(6,4 \%)$.

TABELA 11

Distribuição da população (de fumantes) segundo a presença ou não de sinais e sintomas de distúrbios orgânicos atribuídos ao fumo.

\begin{tabular}{lccccccc}
\hline \multirow{2}{*}{$\begin{array}{l}\text { Presenca } \\
\text { de sinais }\end{array}$} & \multicolumn{3}{c}{ Vinculasão a } & \multicolumn{2}{c}{ Escola } & \multicolumn{2}{c}{ Total } \\
\cline { 2 - 6 } & \multicolumn{2}{c}{ Docentes } & \multicolumn{2}{c}{ Discentes } & Ne & $\%$ \\
\hline não tenho & 17 & 62,9 & 99 & 64,7 & 116 & 64,4 \\
tenho & 10 & 37,1 & 54 & 35,3 & 64 & 25,6 \\
\hline TOTAL & 27 & 100,0 & 153 & 100,0 & 180 & 100,6 \\
\hline
\end{tabular}


Pela Tabela 11 constata-se que a maioria dos docentes $(64,7 \%)$ e dos discentes $(62,9 \%)$ não refere alterações orgânicas relacionadas ao hábito de fumar.

Os docentes e discentes, que referiram alterações citam os problemas respiratórios como os mais freqüentes. Este fato evidencia a coincidência existente entre o sintoma físico mais apresentado e o efeito nocivo mais citado. Tais achados são compativeis ao levantamento feito pela OMS (ROSEMBERG, 1980) quanto à mortalidade proporcional nos fumantes de cigarros em relação aos não fumantes onde o câncer de pulmão, bronquite e enfisema tem os coeficientes mais elevados. Da mesma forma, a associação do fumo com os problemas cardiovasculares, por estar evidenciada nos estudos médicos, foi também enfatizada pelos discentes e docentes (Tabela 10).

\section{TABELA 12}

Distribuição da população fumante segundo a preocupação com os não fumantes a seu redor.

\begin{tabular}{|c|c|c|c|c|c|c|}
\hline \multirow{3}{*}{ Preoc'upacão } & \multicolumn{4}{|c|}{ Vinculação à Escola } & \multirow{2}{*}{\multicolumn{2}{|c|}{$N^{\text {Total }}$}} \\
\hline & \multicolumn{2}{|c|}{ Docentes } & \multicolumn{2}{|c|}{ Discentes } & & \\
\hline & Ne & $\%$ & $\mathbf{N}^{0}$ & $\%$ & & \\
\hline não & $\mathbf{5}$ & 18,5 & 30 & 19,6 & 35 & 19,4 \\
\hline $\operatorname{sim}$ & 22 & 81,5 & 123 & 80,4 & 145 & 80,6 \\
\hline TOTAL & 27 & 100,0 & 153 & 100,0 & 180 & 100,0 \\
\hline
\end{tabular}

Pela Tabela 12 verifica-se que a maioria dos docentes $(81,5 \%)$ e dos discentes $(80,4 \%)$ preocupa-se com a população não fumante a seu redor. O motivo referido mais vezes, por ambos os grupos, como maior fator de preocupação, foi o fato de não estarem respeitando os direitos do próximo. Tanto o grupo de docentes como de discentes que não se preocupa com a população não fumante baseia tal posicionamento nos seguintes argumentos: por se sentirem egoístas e por ser o fumo um hábito muito comum. 
TABELA 13

Distribuição da população conforme orientação dada sobre o fumo às pessoas com as quais se relaciona.

\begin{tabular}{|c|c|c|c|c|c|c|c|c|c|}
\hline \multirow{3}{*}{ Orientação } & \multicolumn{5}{|c|}{ Vinculação à Escola } & & \multicolumn{2}{|c|}{ Total } & \\
\hline & \multicolumn{2}{|c|}{ Docentes } & & \multicolumn{2}{|c|}{ Discentes } & & No & tal & \\
\hline & $\mathbf{N}$ & $\%$ & & $\mathbf{N e}$ & $\%$ & & & & \\
\hline não orienta & 25 & 21,4 & & 244 & 38,4 & & 269 & 35,7 & \\
\hline $\begin{array}{l}\text { orienta em ambiente } \\
\text { profissional }\end{array}$ & 20 & 17,1 & & 76 & 11,9 & & 96 & 12,7 & \\
\hline $\begin{array}{l}\text { orienta em ambiente } \\
\text { extra profissional }\end{array}$ & 6 & 5,1 & $\begin{array}{l}91 \\
77,8\end{array}$ & 23 & 3,7 & $\begin{array}{l}385 \\
60,5\end{array}$ & 29 & 3,9 & $\begin{array}{l}476 \\
63,2\end{array}$ \\
\hline $\begin{array}{l}\text { orienta em qualquer } \\
\text { ambiente }\end{array}$ & 65 & 55,6 & & 286 & 44,9 & & 351 & 46,6 & \\
\hline em branco & 1 & 0,8 & & 7 & 1,1 & & 8 & 1,1 & \\
\hline TOTAL & 117 & 100,0 & & 636 & 100,0 & & 753 & 100,0 & \\
\hline
\end{tabular}

Pela Tabela 13 observa-se que $91(77,8 \%)$ docentes e $385(60,5 \%)$ discentes, perfazendo um total de $476(63,2 \%)$ dão orientações sobre o fumo, sendo essas orientaçōes dadas algumas vezes apenas em ambiente profissional, outras vezes em ambiente extra profissional e para a maior parte $(46,6 \%$ do total) em qualquer ambiente. Nota-se, também, que $269(35,7 \%)$ elementos da população estudada não dão orientações sobre o fumo às pessoas de seu relacionamento. Dentre esses verifica-se que $73(27,1 \%)$ são fumantes e $196(72,9 \%)$ pertencem ao grupo dos não fumantes.

\section{CONCLUSOES}

A vista dos resultados apresentados nas tabelas considera-se que os objetivos foram atingidos, tendo-se chegado às conclusões abaixo descritas.

1. A freqüência de não fumantes predomina em relação aos fumantes e ex-fumantes, tanto entre os docentes como entre os discentes dos cursos de Graduação das Escolas de Enfermagem do Município de São Paulo.

2. As seguintes causas foram enfatizadas como sendo as mais comuns para os 2 grupos, quanto ao fato de:

- iniciar o hábito de fumar: curiosidade e desejo de experimentar o fumo, influência do grupo familiar e ajuda a enfrentar tensões; 
deixar de fumar: autoconscientização sobre os malefícios do fumo para a saúde;

- não fumar: não ter sentido vontade, ter conhecimento dos prejuízos para a saúde, ter experimentado e não ter gostado.

3. A opinião dos dois grupos sobre os efeitos do fumo foram coincidentes; em ambos predominou a opinião de que o fumo traz prejuízos para a saúde, sendo citados como principais efeitos nocivos os relativos a: alterações respiratórias, cardiovasculares, distúrbios orgânicos generalizados, aumento da incidência de câncer e dependência.

4. Mais da metade da população estudada dá orientação sobre o fumo às pessoas de seu relacionamento.

VALENTE, M. A.; MIYADAHIRA, A. M. K.; IDE, C. A. C.; TAKAHASHI, E. I. U.; CHAVES, E. C.; PADILHA, K. G.; KIMURA, M. Smoking in schools of nursing. Rev. Esc. Enf. USP, São Paulo, 16(2):147-163, 1982.

A study was made among students and instructors of three schools of nursing at São Paulo City, Brazil, on number of smokers, non smokers and previous smokers and the causes that led them to start smoking as well as to withdraw from the habit. In a population of 636 studients the authors found $24.1 \%$ smokers, $68.5 \%$ non smokers, $7.4 \%$ previous smokers. The percentage of 117 instructors were $23.1 \%, 62.4 \%$ and $14.5 \%$ respectively. The reason for start smolking most frequently mentioned by both groups was curiosity $(35.2 \%$ and $28.2 \%$ respectively). For leaving the habit was conscience of damage to health $(72.5 \%$ and $65.2 \%)$. For the non smokers this was the main reason for never starting the habit. The authors studied also the age of start smoking, number of cigarettes smoked per day. The outside influences for smoking or not orientation of others about the damages of smoking to health were also considered.

\section{REFERÊINCIAS BIBLIOGRÄFICAS}

1. ANGElli, M. Cigarro, a riqueza nacional que mata. O Estado de São Paulo, São Paulo, 17 março 1981, c. 1, p. 16.

2. GODBER, G. Libelo contra o fumo. Saúde Mundo, Grenebra, 23(2):20-5, fev./mar., 1970.

3. GRY, J. A. M. Warning: cigarettes may do you good smoking has beneficial effects for some because its helps them to cope mere effectively with stress. Nurs. Mirror, SusseX, 150(15):21-3, 10 Apr., 1980.

4. HORN, D. As razões do fumar. Saúde Mundo, Genebra, dez. 1975, p. 26-31.

5. ORganización mundial IE LA SALUd. Fumo ou saúde: a escolha é sua. Washington, 1980. p. 29. (Publicação avulsa - Dia Mundial da Saúde -7 de abril de 1980).

6. RESOURCE gulde on smoking and health for canadian schools. 2. ed. s.l.p., The Minister of National Health and Welfare, 1976. 43p.

7. Rosemberg, J. Tabagismo: sério problema de saúde pública. São Paulo, Santos 1977. 236p.

8. - Métcdos de controle da epidemia tabagista. Rev. Div. Nac. Pneumol. Sanit., Rio de Janeiro, 24(93):1-16, jan./mar., 1980.

9. SCHURER, M. Smoking and the consequences. Nurs. Times, London, 76(11):565-8, 3 Apr., 1980.

10. Williard, N. O dragão do fumo, obra do homem. Saúdo Mundo, Genebra, 32(5):4-9, June 1979. 


\section{ANEXO I}

Pesquisa sobre o Hábito de Fumar

$N^{9}$ Descrição da Variável

1. Número do questionário

2. Escola

(0) Escola de Enfermagem U.S.P.

(1) Escola Paulista de Enfermagem

(2) Escola São José

3. Tipo de vinculação com a Escola:
(0) docente
(1) aluno $1^{\circ}$ semestre
(2) " $" 2$ "
(3) " 3 " "
(4) " $44^{\circ} "$
(5) $" 5^{\circ} "$
(6) " $" 6^{\circ} \quad "$
(7) $" 7^{\circ} "$
(8) " 8" "

4. Sexo:

(0) Masculino

(1) Feminino

5. Idade:

(0) Menos de 20 anos

(1) 20 a 29 anos

(2) 30 a 39 anos

(3) 40 a 49 anos

(4) 50 anos ou mais

6. Com que idade começou a fumar?

(0) nunca comecei a fumar

(1) antes dos 10 anos

(2) 10 a 14 anos

(3) 15 a 1.9 anos

(4) 20 a 24 anos

(5) 25 a 29 anos

(6) 30 anos ou mais

7. Há quanto tempo fuma?
(0) nunca fumei
(1) deixei de fumar
(2) menos de 1 ano 

(3) 1 a 2 anos
(4) 3 a 4 anos
(5) 5 a 6 anos
(6) 7 a 8 anos
(7) 9 a 10 anos
(8) 11 a 15 anos
(9) mais de 15 anos

8. Quantos cigarros fuma por dia?
(0) nenhum
(1) 1 a 5 cigarros
(2) 6 a 10 cigarros
(3) 11 a 15 cigarros
(4) 16 a 20 cigarros
(5) 21 a 25 cigarros
(6) 26 a 30 cigarros
(7) mais de 30 cigarros

9. Há quanto tempo deixou de fumar?
(0) nunca fumei
(1) nunca deixei de fumar
(2) menos de 1 ano
(3) 1 a 2 anos
(4) 3 a 4 anos
(5) 4 a 5 anos
(6) 6 anos ou mais

10. Durante quanto tempo fumou?

(0) nunca fumei

(1) nunca deixei de fumar

(2) menos de 1 ano

(3) 1 a 2 anos

(4) 3 a 4 anos

(5) 5 a 6 anos

(6) 7 a 8 anos

(7) 9 a 10 anos

(8) 11 anos ou mais

11. Por que começou a fumar?

(Indique, no máximo, 3 opções)

(0) nunca comecei a fumar

(1) influência do grupo familiar

(2) influência do grupo extra familiar

(3) curiosidade e desejo de experimentar o fumo

(4) ajuda a enfrentar tensões e estudos

(5) sentir-se adulto 

(6) aumenta o atrativo pessoal
(7) desconhecimento dos prejuizos à saúde
(8) não sabe
(9) outros:

12. Por que deixou de fumar?

(0) nunca fumei

(1) nunca deixei de fumar

(2) deixei de fumar porque:

13. Por que você não ingressou no tabagismo?

(Indique, no máximo, 3 opções)

(0) sou tabägista

(1) nunca senti vontade de fumar

(2) experimentei mas não gostei

(3) tenho conhecimento dos prejuizos à saúde ( )

(4) por influência do grupo familiar ( )

(5) por influência do grupo extra familiar ( )

(6) por problemas de saúde anterior ( )

(7) não sei

(8) outros motivos: ( )

14. Quais os efeitos qua vocè acha que o fumo traz à saúde?
(0) não traz efeitos
(1) $\operatorname{traz} o(\mathrm{~s})$ seguinte(s) efeito(s):

15. Tem apresentado algum sinal ou sintomas que atribue ao fumo?

(0) nunca fumei

(1) não fumo atualmente

(2) não tenho apresentado sinais e/ou sintomas

(3) tenho apresentado os seguintes sinais e/ou sintomas 
16. Quando você fuma, preocupa-se com os não fumantes ao seu redor?

(0) não sou fumante

(1) não me preocupo Porque:

(2) preocupo-me Porque:

17. Você orienta as pessoas de seu relacionamento sobre o fumo?

(0) não oriento

(1) só oriento em ambiente profissional

(2) só oriento em ambiente extra profissional

(3) oriento em qualquer ambiente

\section{ANEXO II}

São Paulo, ......... de de 1981.

Sr. Docente

O grupo de docentes de Enfermagem Médico-Cirúrgica I da EEUSP está fazendo uma pesquisa sobre o hábito de fumar entre professores e alunos de Enfermagem. Tal estudo foi motivado pela alta freqüência de fumantes entre enfermeiros, constatada tanto pela observação direta como através da literatura especializada.

Solicita-se, portanto, a preciosa colaboração de todos para que esse trabalho possa chegar a termo o mais breve possivel.

Antecipadamente, agradece.

Maria Aparecida Valente Responsável pela disciplina E M C I da EE USP

Entregue em .........................

Solicita-se devolução até 